\section{IDDF2019-ABS-0289 A CIRCRNA SIGNATURE PREDICTS POSTOPERATIVE RECURRENCE IN STAGE II/ III COLON CANCER}

${ }^{1}$ Huai-Qiang Ju*, 'Qi Zhao, ${ }^{1}$ Feng Wang, ${ }^{2}$ Ping Lan, 'Zixian Wang, ${ }^{1}$ Rui-Hua Xu. ${ }^{1}$ State Key Laboratory of Oncology in South China, Collaborative Innovation Center for Cancer Medicine, Sun Yat-sen University Cancer Center, China; ${ }^{2}$ The Sixth Affiliated Hospital, Sun Yat-sen University, China

\subsection{6/gutjinl-2019-IDDFabstracts.60}

Background Current staging methods seem to have only a limited role in predicting the risk of disease recurrence and benefit of adjuvant chemotherapy for patients with stage II/III colon cancer. Circular RNA is a novel type of noncoding RNA with a potential use as biomarkers; however, whether circRNA-based signatures could serve as novel prognostic biomarkers for stage II/III colon cancer is unknown.

Methods 20 paired of frozen tumor tissues and adjacent normal tissues of stage II/III colon cancer were collected and conducted an RNA-sequencing study and profiled circRNAs by a series of bioinformatics analyses to identify the significant circRNA markers. QPCR assay was used to test those markers on the samples from the training and validation cohorts. LASSO-bagging procedure was used to select the top four markers to build the regression model. The cell migration assay in vitro and metastasis study in vivo were performed to detect the function of the top four markers.

Results Dysregulated circRNAs showed strong classification properties in distinguishing the recurrent and nonrecurrent colon cancer patients. A novel prognostic tool (cirScore) based on four circRNAs (i.e., hsa_circ_0122319, hsa_circ_0087391, hsa_circ_0079480 and hsa_circ_0008039) is developed and validate $\bar{d}$ to improve the prognostic stratification for patients with radically resected stage II/III colon cancer. The proposed cirScore can effectively classify patients with stage II/III colon cancer into groups with low and high risks of disease recurrence. Loss-of-function assays indicated that the representative circRNAs plays functional roles in the sophisticated regulation of colon cancer progression.

Conclusions Our current study addresses an important gap, which is the refinement of our prognostic tools for stage II/ III colon cancer, by using a novel approach that takes into consideration the circular RNA. The proposed cirScore might be used in the future to guide better and more personalized treatment decisions for patients with stage II/III colon cancer.

\section{IDDF2019-ABS-0290 IGF2BP2 FACILITATES TUMOR PROGRESSION VIA AN M6A-DEPENDENT MECHANISM IN COLORECTAL CARCINOMA}

Ting Li*, Pei-Shan Hu, Jin-Fei Lin, Huai-Qiang Ju, Rui-Hua Xu. State Key Laboratory of Oncology in South China, Collaborative Innovation Center for Cancer Medicine, Sun Yat-sen University Cancer Center, China

\subsection{6/gutjnl-2019-IDDFabstracts.61}

Background RNA N6-methyladenosine (m6A) is an emerging regulatory mechanism for gene expression and participates in tumor progression in several cancer types. M6A 'readers' were reported to be involved in controlling the fate of mRNA, and insulin-like growth factor 2 mRNA binding protein 2 (IGF2BP2) was reported to be associated with methylated mRNA stability and promote tumor progress However, its role in colorectal carcinoma (CRC) and its target m6A-specific target genes remains unexplored.

Methods Western blot, real-time quantitative PCR and immunohistochemical (IHC) analysis were used to detect IGF2BP2 expression in cell lines and patient's tissues. The MTS assay, migration assay, sphere formation assay was performed to detect the function of IGF2BP2. Cell-based xenograft model and PDX model revealed the clinical benefits of target IGF2BP2 in vivo. RNA immunoprecipitation (RIP) sequence was used to screen the target genes of IGF2BP2 and RNA pull-down assay was used to verify the direct binding of IGF2BP2 and targets genes.

Results IGF2BP2 was significantly upregulated in human CRC tumor tissues and CRC cell lines and high expression of IGF2BP2 was associated with poor prognosis of CRC patients. Knockdown of IGF2BP2 in CRC cell lines drastically suppressed cellular proliferation and stemness phenotype in vitro. IGF2BP2 inhibition suppresses CRC tumorigenesis and metastasis in both cell models and PDX models in vivo. RIP-seq analysis and RIP-qPCR revealed that SOX2 mRNA was the potential target gene of IGF2BP2. RNA pull-down assay showed the direct binding of IGF2BP2 and SOX2 transcripts which could be impaired by mutating m6a site of $S O X 2$ transcripts. After IGF2BP2 inhibition, the RNA and protein level of SOX2 were downregulated due to the half-life of SOX2 mRNA decreased. Moreover, the downstream genes of SOX2 showed a positive correlation with IGF2BP2 expression in CRC patients.

Conclusions In summary, we demonstrated that IGF2BP2 was frequently upregulated in human CRC and contributing to CRC malignancy. IGF2BP2 maintained methylated SOX2 mRNA stability to facilitate cellular stemness features through the m6A-dependent mechanism. Thus, our findings reveal an important role of IGF2BP2 and provide a potential target of treatment in colorectal carcinoma.

\section{IDDF2019-ABS-0292 NUCLEUS-TRANSLOCATED GCLM FACILITATES TUMOR PROGRESSION THROUGH INCREASING TRANSCRIPTION OF OCT4 IN COLORECTAL CARCINOMA}

Jin-Fei Lin*, Pei-Shan Hu, Ting Li, Huai-Qiang Ju, Rui-Hua Xu. State Key Laboratory of Oncology in South China, Collaborative Innovation Center for Cancer Medicine, Sun Yat-sen University Cancer Center, China

\subsection{6/gutjnl-2019-IDDFabstracts.62}

Background Glutamate-cysteine ligase modifier (GCLM) subunits, combined with catalytic (GCLC) subunits, has been known for the first rate-limiting enzyme for glutathione (GSH) synthesis. Increasing studies have shown that the nonmetabolic functions of many metabolic enzymes also play a crucial role in tumor progress. However, the non-metabolic functions of GCLM remain unexplored. Hence, we wonder if there are unorthodox functions of GCLM under redox stress participating colorectal Carcinoma(CRC) tumorigenesis.

Methods Western blot, real-time quantitative PCR and immunohistochemical analysis were used to detect the relative expression of GCLM in cell lines and patients' specimens. The MTS assay, migration and invasion assay, sphere formation assay were performed to detect the functions of GCLM in vitro, and the xenograft models and PDX models in nude mice in vivo. Cytosolic and nuclear extraction, immunofluorescent analysis were used to show the location of GCLM. 
Chromatin immunoprecipitation (ChIP) assay was used to screen and verify the GCLM target genes.

Results We found that glucose deprivation condition upregulated the expression of GCLM rather than hypoxia or $\mathrm{H} 2 \mathrm{O} 2$. Compared to adjacent tumor tissues, the expression of GCLM was upregulated in CRC tumor tissues. The high expression of GCLM predicted poor prognosis in CRC patients. Inhibition of GCLM results in decreased proliferation rate, migration and invasion ability and sphere formation in HCT116 and DLD1 in vitro. Similarly, GCLM inhibition suppressed CRC tumorigenesis and metastasis in xenograft models and PDX models in vivo. The cancer cell stem cell markers were also downregulated after GCLM inhibition. Interestingly, we found that glucose starvation could rescue the anti-cancer phenotypes of GCLM inhibition and led to nucleus-translocation and accumulation of GCLM. ChIP assay showed GCLM could interact with octamer-binding transcription factor 4 (OCT4) promoter, a cancer stem cell marker, to increase its expression, and the target gene of OCT4 showed a positive correlation with GCLM expression in CRC patients.

Conclusions Our data showed that GCLM displayed nucleus accumulation in the condition of glucose deprivation which increased the transcription of OCT4. This study revealed an unorthodox oncogenic function of GCLM in colorectal carcinoma and suggested GCLM as a potential therapeutic target in CRC.

\section{IDDF2019-ABS-0293 A MICROBIOME PILOT STUDY: THE EXPLORATION OF THE GUT-SKIN AXIS IN HIDRADENITIS SUPPURATIVA}

\begin{abstract}
${ }^{1}$ Suk Yee Lam*, ${ }^{2}$ Djawad Radjabzadeh, ${ }^{1}$ Hester Eppinga, ${ }^{3}$ Hessel $\mathrm{H}$ van der Zee, ${ }^{2}$ Robert Kraaij, ${ }^{1}$ Sergey R Konstantinov, ${ }^{1}$ Gwenny M Fuhler, ${ }^{3}$ Errol P Prens, ${ }^{3}$ Hok Bing Thio, ${ }^{1}$ Maikel P Peppelenbosch. 'Department of Gastroenterology and Hepatology, Erasmus University Medical Center, Netherlands; ${ }^{2}$ Department of Internal Medicine, Erasmus University Medical Center, Netherlands; ${ }^{3}$ Department of Dermatology, Erasmus University Medical Center, Netherlands
\end{abstract}

\subsection{6/gutjnl-2019-IDDFabstracts.63}

Background The concurrent occurrence of gut and skin diseases has led to the postulation of the existence of a gut-skin axis in which gut microbiota is thought to contribute to extra-intestinal skin manifestations. A dysbiotic skin microbiome has been demonstrated in the chronic inflammatory skin disease hidradenitis suppurativa (HS), but it remains unknown whether microbial disturbances of the gut are engaged in the complex pathogenesis. This study investigates the fecal microbiome in HS patients and healthy controls and explores the possibility of a gut-skin axis in HS.

Methods A total of 17 HS patients and 20 healthy controls provided a stool sample for $16 \mathrm{~S}$ ribosomal RNA (rRNA) gene sequencing. A subset of axillary skin samples was available for concurrent microbial analysis of HS lesions $(n=6)$, paired nonlesions $(n=3)$ and healthy skin of controls $(n=7)$. No oral antibiotics and no topical antibiotics/steroids were used in respectively eight weeks and seven days prior to sample collection.

Results The majority of 16S rRNA gene sequences at both the fecal and axillary skin sites were assigned to phyla Bacteroidetes, Firmicutes, Proteobacteria and Actinobacteria. Based on the overall bacterial community, two distinct clusters could be distinguished between the gut and the skin using principal coordinates analysis (PCoA). Furthermore, significant differences in bacterial composition were observed in fecal samples of HS patients when compared to healthy controls. However, we did not detect altered microbial signatures in the skin samples taken from HS lesions, non-lesions, and healthy controls.

Conclusions These findings indicate the presence of a distinct fecal microbiome in HS patients compared to healthy controls, suggesting the involvement of a gut-skin axis in the etiology of HS. Dysbiosis of the cutaneous microbiome in HS could not be confirmed here, but larger studies are warranted to further elucidate the role of microbiota as a potential part of the gut-skin axis in HS.

\section{IDDF2019-ABS-0294 HSF2 PROMOTE THE MUCOSAL REPAIR IN ULCERATIVE COLITIS BY INHIBITING PRO- INFLAMMATORY CYTOKINE AND PROMOTING TGF- $\beta$ EXPRESSION}

JunKun Niu*. Department of Gastroenterology, The First Affiliated Hospital of Kunming Medical University, China

\subsection{6/gutjnl-2019-IDDFabstracts.64}

Background In our previous study, we found that fecal heat shock transcription factor 2 (HSF2) concentration may be an evaluation index for predicting the mucosal healing of Ulcerative Colitis (UC). So this study is aiming to explore the mechanism of HSF2 in the mucosal repair of UC.

Methods The DAI, colon damage score and histopathology score were recorded in DSS induced colitis. The transcription and expression level of HSF2 and TGF- $\beta$ in the mice colon tissue were detected by PCR and immunohistochemistry. HSF2 levels in HT-29 cells was manipulated by RNA interference and plasmids transfection. The concentrations of IL-1 $\beta$, TNF$\alpha$ and TGF- $\beta$ in cells supernatant were detected by ELISA after induced by LPS. The phosphorylation level of ERK, P38, JNK and $S$ mad2/3 were detected by Western Blot.

Results The DAI, colon damage and pathology score of methylprednisolone(MP)treatment group were lower than DSS model group (figure 1). The transcription and expression of HSF 2 and TGF- $\beta$ in colon mucosal tissue were increased and decreased synchronously in DSS model group and MP treatment group(figure 2). The phosphorylation level of ERK, P38 and JNK increased significantly in HSF2 siRNA compared with the negative control group. The concentrations of IL-1 $\beta$ and TNF- $\alpha$ in the supernatant of HT-29 cells increased in HSF2 siRNA group. On the contrary, the phosphorylation level of ERK, P38 and JNK decreased significantly in HSF2FLAG plasmid transfection compared with the Blank Vector group. The concentrations of IL- $1 \beta$ and TNF- $\alpha$ in the supernatant of HT-29 cells reduced (figure 3 , figure 4 ).

The phosphorylation level of Smad2/3 decreased in HSF2 siRNA compared with the negative control group. The concentrations of TGF- $\beta$ in the supernatant of HT-29 cells reduced in HSF2 siRNA group. On the contrary, the phosphorylation level of Smad2/3 increased significantly in HSF2FLAG plasmid transfection compared with the Blank Vector group. The concentrations of TGF- $\beta$ in the supernatant of HT-29 cells increased (Figure 4).

Conclusions HSF2 can promote the mucosal repair of ulcerative colitis by inhibiting the inflammatory response and promoting mucosal repair factor expression. 